Int. J. Dev. Biol. 57: 759-765 (2013)

doi: $10.1387 / \mathrm{ijdb} .130158 \mathrm{ab}$

\title{
Eph receptors and ephrin class B ligands are expressed at tissue boundaries in Hydra vulgaris
}

\author{
SUSANNE TISCHER ${ }^{1}$, MONA REINECK ${ }^{1}$, JOHANNES SÖDING ${ }^{2}$, SANDRA MÜNDER ${ }^{1}$ and ANGELIKA BÖTTGER ${ }^{*, 1}$ \\ ${ }^{1}$ Department of Biology 2, Ludwig-Maximilians-Universität München, ${ }^{2}$ Gene Center Munich and Department of \\ Biochemistry, Ludwig-Maximilians-Universität München, Munich, Germany
}

\begin{abstract}
Eph receptors and ephrins are important players in axon guidance, cell sorting and boundary formation. Both the receptors and the ligands are integrated transmembrane proteins and signalling is bidirectional. The prevalent outcome of signal transduction is repulsion of adjacent cells or cell populations. Eph/ephrins have been identified in all multicellular animals from human to sponge, their functions however appear to have been altered during evolution. Here we have identified four Eph receptors and three class B ligands in the cnidarian Hydra vulgaris, indicating that those are the evolutionary older ones. In situ hybridisation experiments revealed a striking complementarity of expression of receptors and ligands in tentacles and in developing buds. This suggests that the original function of ephrin signalling may have been in epithelial cell adhesion and the formation of tissue boundaries.
\end{abstract}

KEY WORDS: Eph-receptor, ephrin, hydra, boundaries, phylogeny

Eph receptors and ephrins are both integral transmembrane proteins and need cell-cell contact for activation. They interact with cell-surface-molecules, for example Notch (Limbourg et al., 2007), adhesion molecules like integrins and E-cadherins (Orsulic and Kemler, 2000) and components of the Wnt-signalling pathway (Batlle et al., 2002). Their downstream effectors are in most cases regulators of the cytoskeleton and/or of cell adhesion. They regulate cell sorting at boundaries, changes of cell adhesion to the extracellular matrix and alterations of cell morphology. EphRs/ephrins are prominently involved in developmental processes within the nervous system including axon guidance, growth cone spreading of motor neurons and dendritic spine morphogenesis (Kao et al., 2012).

Ephrin and EphR sequences are strongly conserved throughout the animal kingdom. Drosophila and Caenorhabditis elegans only have one EphR each, whereas vertebrates possess 15. In C.elegans they function in early embryonic morphogenetic events, e.g. closure of the gastrulation cleft (reviewed in (Simske and Hardin, 2001)). They also play a role in axon targeting in the nervous system (Mohamed and Chin-Sang, 2006). Work in Drosophila has focussed on the role of EphR/ephrin signalling for neural development and synaptic function. This is also a major theme in vertebrates, where ephrins are involved in topographic mapping of neuronal connections (Knöll and Drescher, 2002). It was suggested, that with increasing size of the Eph-family, new functions could have been adopted (Drescher, 2002).
Hydra vulgaris is a small freshwater polyp belonging to the phylum of cnidaria and thus evolved before bilaterians split off from the early metazoan phyla. Hydra is a simply built animal, consisting of a body column with a posterior foot structure and a head at the apical side. Hydra has few visible tissue boundaries, including one between the tentacle zone and the tentacles, one between the peduncle and the basal disc and one that separates the bud from the parent animal at the end of the budding process, which is Hydras way of asexual reproduction (Böttger and Hassel, 2012). The diffuse cnidarian nervous system with multifunctional neurons is arranged in a net like structure with some condensed areas and probably represents an ancestral form in nervous system evolution (Grimmelikhuijzen and Westfall, 1995). Many major developmental signalling pathways, including Notch (Käsbauer et al., 2007) and BMP (Reinhardt et al., 2004) have been identified in Hydra indicating an early origin of metazoan signalling modules.

We have now analysed the occurrence of EphR/ephrins in Hydra. We found that four genes encoding EphRs and three encoding class B ephrins are present, indicating an unexpected complexity. We show that these genes are expressed in tentacles and buds, and some of them mark the respective boundaries. We

Abbreviations used in this paper: EphR, Eph receptor; GPI, glycosylphosphatidylinositol; RTK, receptor tyrosine kinase; TyrK, tyrosine kinase; SAM, sterile alpha motif.

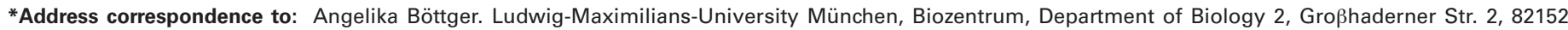
Planegg-Martinsried, Munich, Germany.Tel: 0049-89-218074-279. Fax: 0049-89-218074-219. E-mail: boettger@zi.biologie.uni-muenchen.de http://www.cellbiology.bio.Imu.de/research_groups/boettger/index.html
} 


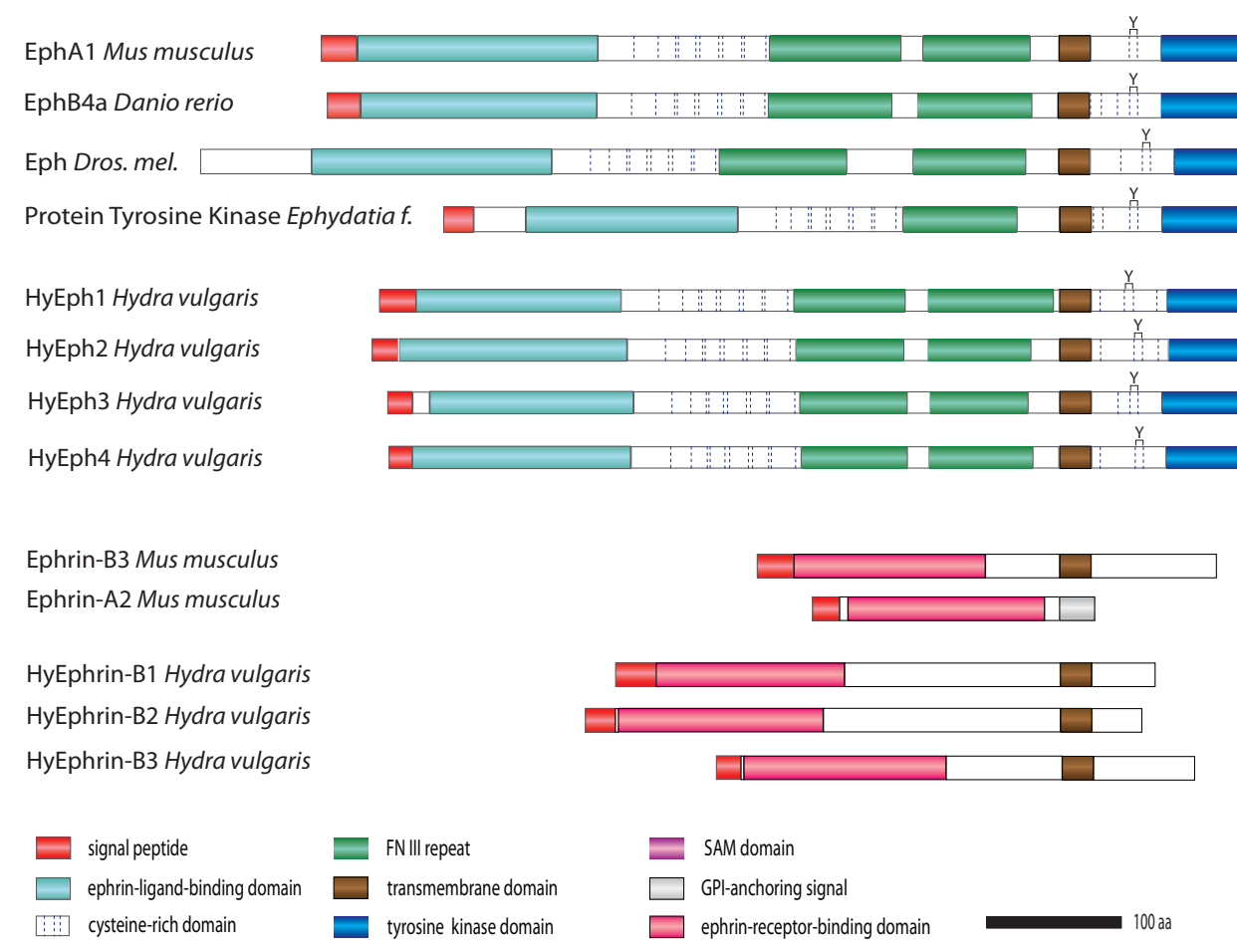

Fig. 1. Schematic representation of the protein domain structure of different Eph receptors and ephrin ligands. Included are signal peptide (color: red), ephrin-ligand-binding domain (EPH-Ibd) (light blue), cysteine-rich domain (dashed lines), fibronectin type III (FN III) repeats (green), transmembrane domaine (brown), tyrosine kinase domain (dark blue), sterile-alpha-motif (SAM) (purple), ephrin-receptor-binding domain (RBD) (pink) and GPI-linkage signal (grey). Mm, Mus musculus; Dr, Danio rerio; Dm, Drosophila melanogaster; Ce, C. elegans; Hv, Hydra vulgaris; Ef, Ephydatia fluviatilis. GenBank accession numbers: Receptors: Mus musculus EphA1, (NP_076069.2); Danio rerio EphB4a, (NP_001005919); Drosophila melanogaster Eph, (NP_726589); Ephydatia fluviatilis (sponge) Protein Tyrosine Kinase, (BAA81719.3); Hydra vulgaris HyEph1, (KC751429); Hydra vulgaris HyEph2, (KC751430); Hydra vulgaris HyEph3, (KC751432); Hydra vulgaris HyEph4, (KC751431). Ligands: Mus musculus Ephrin-B3, (EDL 10502.1); Mus musculus Ephrin-A2, (EDL31583.1); Hydra vulgaris HyEphrin-B1, (KC751433); Hydra vulgaris HyEphrin-B2, (KC751434); Hydra vulgaris HyEphrin-B3, (KC751435).

thus speculate that this cell-contact mediated signalling pathway originally evolved to regulate cell adhesion and cell contacts at simple tissue boundaries.

\section{Results and Discussion}

\section{Cloning of EphRs and Ephrins}

Sequences encoding four different Eph receptors (EphRs) and three class-B-ligands were identified in the Hydra genome. We cloned full length cDNAs representing these sequences from Hydra vulgaris (strain Basel). The receptors were named HyEph1, $-2,-3$, and -4 . HyEph2 contained the complete sequence of one of three recently published fragments for putative Hydra EphRs (Hma1.122109), HyEph4 contained both sequences of the other two published fragments (Hma1.108443, Hma1.109586), indicating that they both belong to the same gene (Reddy et al., 2011). We found one fragment of an additional putative Hydra EphR, for which we have not yet isolated a full length cDNA (HyEph5, partial (Genbank accession number: KC788277) data not shown).

Ephrin ligands are distinguished into ephrinAs and ephrinBs, according to their domain structure, which involves a C-terminal GPI-anchoring signal for ephrinAs. The three ephrins from Hydra are thus clearly ephrin B type (Fig. 1). They were named HyEphrinB1, HyEphrin-B2 and HyEphrin-B3 (according to the nomenclature committee (https://eph-nomenclature.med.harvard.edu)).
The conserved EphR domain structure is found in all of our HyEphRs (Smalla et al., 1999) (Fig. 1). The tyrosine residues required for autoactivation of the activation loops within the RTKdomains are also conserved. Furthermore, two conserved tyrosine residues within the motif $Y X X X X X Y$ in the juxtamembrane region are present, which in mammalian receptors present a platform for interactions with $\mathrm{SH}-2$ domains (reviewed in (Himanen and Nikolov, 2003)). We therefore expect a similar activation mode for the Hydra EphRs as in mammals (Fig. 1).

EphRs are classified either as class A or B receptors, according to which kind of ligands they bind to. It is not possible to distinguish between them based only on sequence comparisons. As we are lacking interaction data at the moment, we did not classify the Hydra receptors. Nevertheless we expect them to be class $B$ receptors, accounting for the fact, that we have three class B ligands in Hydra, but not a single class A ligand.

\section{Phylogeny}

For phylogenetic analysis neighbour joining trees were calculated (Fig. 2A,B). Protein sequences of the HyEphRs and HyEphrins were compared with those of vertebrates, urochordates, echinodermates, nematodes, insects and one available sequence of porifera. We only included sequences encoding predicted full length EphRs and ephrins. In case of the EphRs the sequences of the tyrosine kinase domains were analysed (TyrKs). The TyrK-domain of the mouse 
A

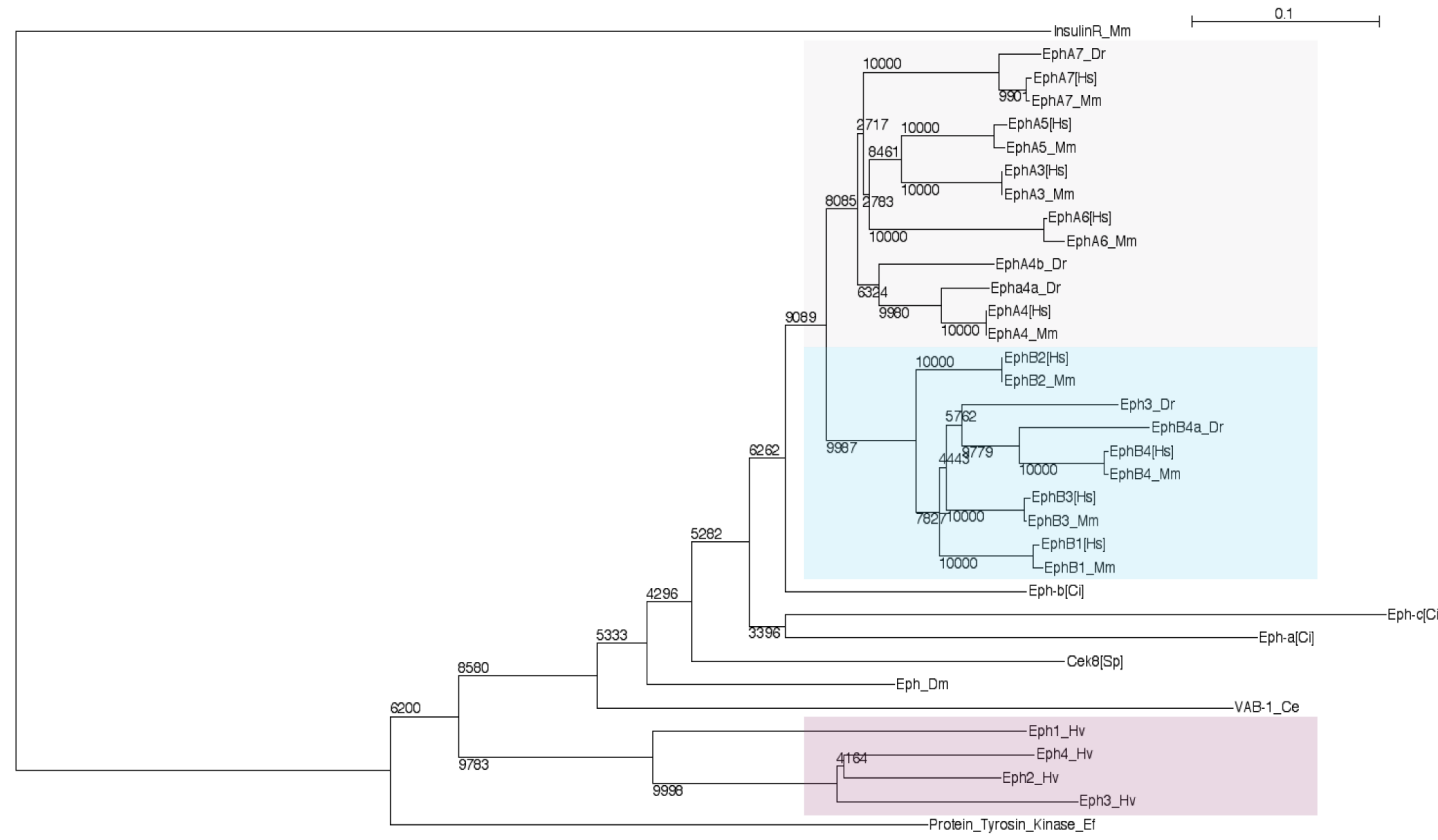

B

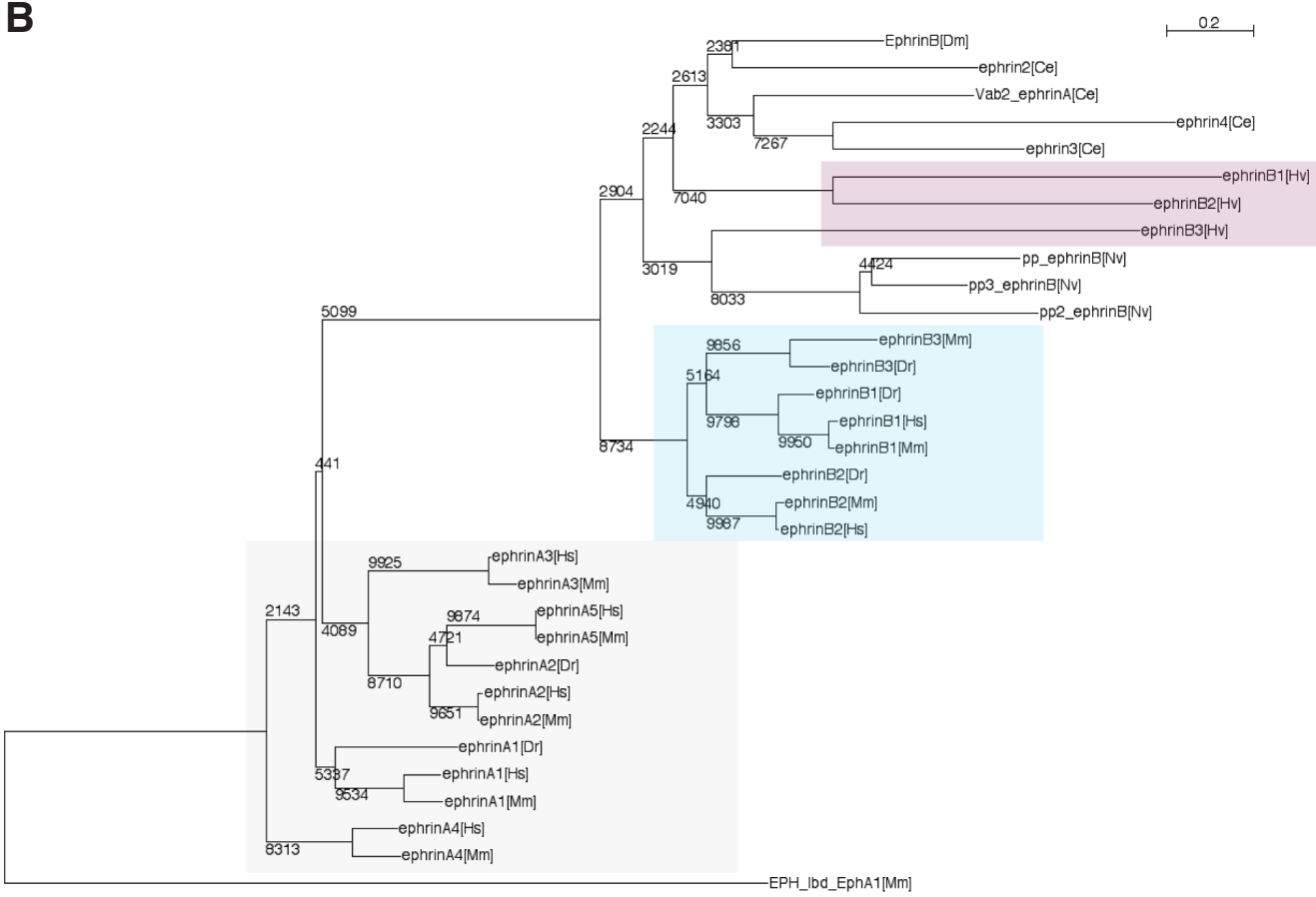

Fig. 2. Phylogenetic analysis. NJ trees on the basis of a ClustalX alignments of the amino acid sequences. Hs: Homo sapiens, Mm: Mus musculus, Dr: Danio rerio, $S p$ : Strongylocentrotus purpuratus, Dm:Drosophila Melanogaster Ci: Ciona intestinalis, Ce: C. elegans, Hy: Hydra vulgaris, Ef: Ephydatia fluviatilis. (A) Tyrosine kinase domains have been used for analysis of the Eph receptors. The Insulin-receptor tyrosine-kinase domain of Mus musculus was used as outgroup. EphAs are encircled in grey, EphBs in turquoise. Hydra EphRs are highlighted in purple. (B) Receptor binding domains (RBDs) have been used for analysis of the ephrin ligands. The Ephrin-ligand binding domain (EPH-Ibd) of EphA1-receptor Mus musculus was used as outgroup. Vertebrate class A ligands are encircled in grey, vertebrate class $B$ ligands in turquoise. Hydra ephrins are highlighted in purple. GenBank accession numbers (A): Homo sapiens HsEphA3 (NP_005224.2), HsEphA4 (NP_004429.1), HsEphA5 (NP_004430.3), HsEphA6 (Q9UF33), HsEphB1 (NP_004432.1), HsEphB2 (NP_004433.2), HsEphB3 (NP_004434.2), HsEphB4 (NP_004435.3); Mus musculus EphA3 (NP_034270), EphA4 (NP_031962), EphA5 (NP_031963), EphA6 (NP_031964), EphA7 (NP_034271), EphB1 (NP_775623), EphB2 (NP_034272), EphB3 (NP_034273), EphB4 (NP_034274); Danio rerio Eph receptor Eph3 (AAK54726.1), EphA4a (NP_001005919), EphA4b (AAK54725), EphA7 (NP_001038444), EphB4a

(NP_571489); Strongylocentrotus purpuratus SpCek8 (XP_001199846.1); Drosophila melanogaster Eph (NP_726589); Caenorhabditis elegans VAB-1 (NP_494806); Ephydatia fluviatilis Protein Tyrosine Kinase (BAA81719.3); Ciona intestinalis CiEph-a (BAE06400.1), CiEph-b (BAE06403.1), CiEph-C (NP_001071691.1); Hydra vulgaris HyEph1 (KC751429), HyEph2 (KC751430), HyEph3 (KC751432), HyEph4 (KC751431). GenBank accession numbers (B): Homo sapiens ephrinA1 (NP_004419.2), ephrinA2 (NP_001396.2), ephrinA3 (NP_004943.1), ephrinA4 (NP_005218.1), ephrinA5 (NP_001953.1), ephrinB1 (NP_004420.1), ephrinB2 (AAH74856.1); Mus musculus ephrinA1 (EDL15212.1), ephrinA2 (EDL31583.1), ephrinA3 (AAI25004.1), ephrinA4 (EDL15210.1), ephrinA5 (AAH40218.1), ephrinB1 (AAH21656.1), ephrinB2 (AAH57009.1), ephrinB3 (EDL10502.1); Drosophila melanogaster ephrinB (AAF28394.1); Caenorhabditis elegans VAB-2 (CCD74044.1), efn-2 (NP_501955), efn-3 (NP_510250), efn-4 (NP_499947); Danio rerio ephrin-A1 (AAH56770.1), efna2 (NP_571097), ephrin-B1 (AAK64274.1), EphrinB2 (NP_571098.1), EfnB3 (AAH95605.1); Nematostella vectensis predicted protein (EDO47804.1), predicted protein2 (EDO44950.1), predicted protein3 (EDO36327.1); Hydra vulgaris HyEphrin-B1 (KC751433), HyEphrin-B2 (KC751434), HyEphrin-B3 (KC751435) 

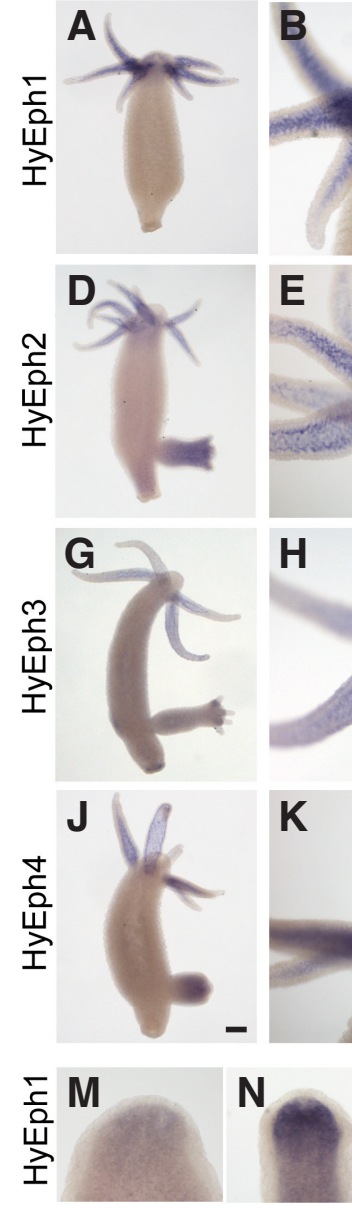
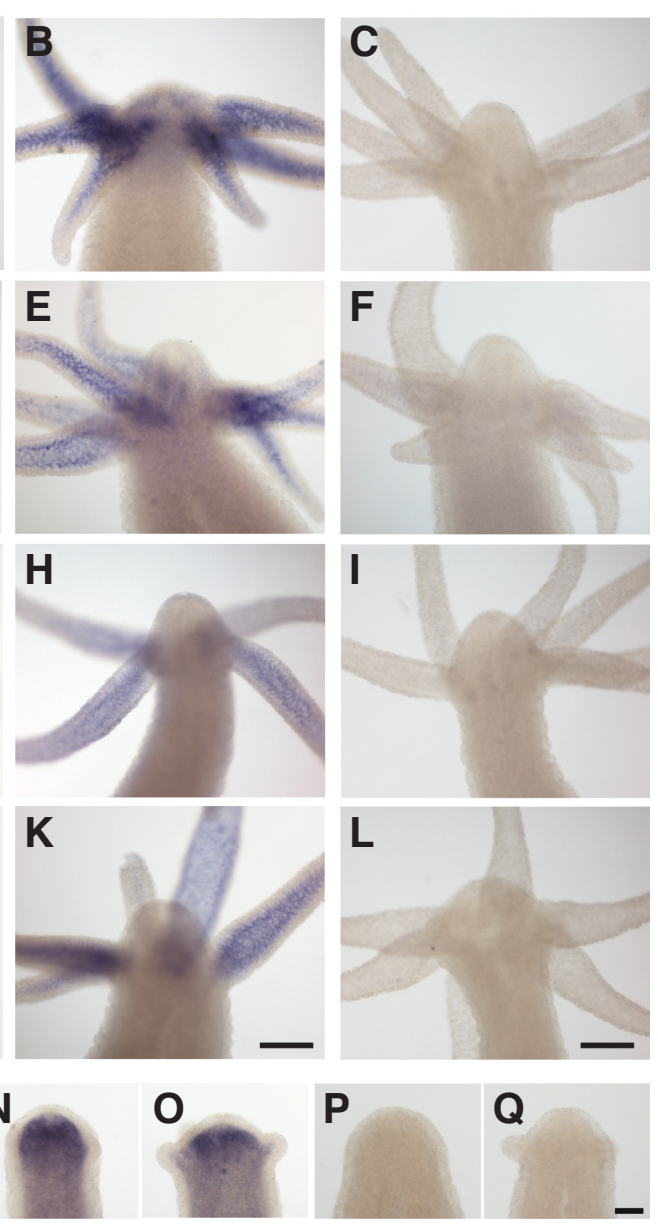
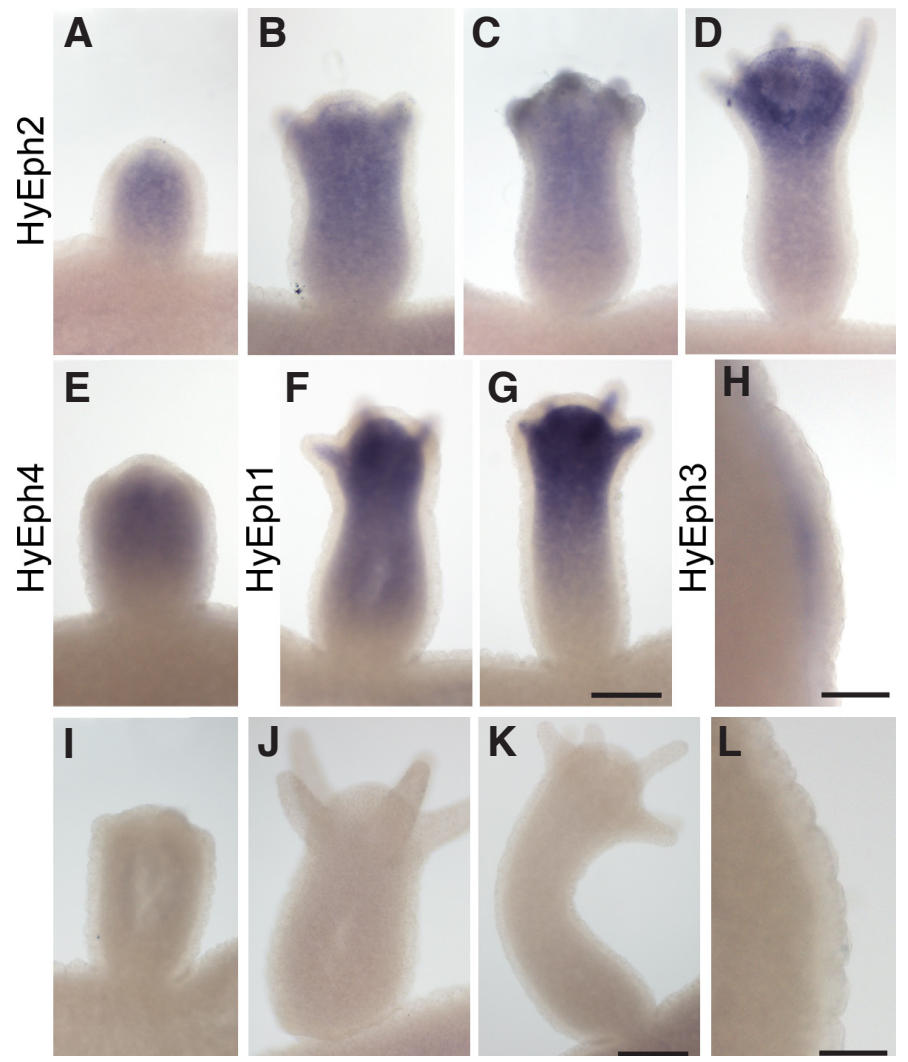

Fig. 3 (left). Whole-mount in situ hybridisation for the Eph receptors HyEph1, HyEph2, HyEph3 and HyEph4 on adult and budding animals (A$\mathrm{L}$ ) and for HyEph1 during different stages of head regeneration (M-Q) (24 hours (panel M) and 36 to 48 hours (panels $\mathbf{N}, \mathbf{0})$. NBT/BC/P (Roche) was used for staining reactions (blue signals). (A, B,D,E, G, H, J,K, M, N,O) anti-sense; (C,F,I,L,P,Q), sense. Scale bars: (A-L), $100 \mu \mathrm{m}$; (M-Q) $50 \mu \mathrm{m}$.

Fig. 4 (right). Whole-mount in situ hybridisation for HyEph1, HyEph2, HyEph3, HyEph4 on developing buds. NBT/BCIP (Roche) waS used for staining reactions (blue signals). (A-H) anti-sense; (I-L) sense (I-K, HyEph2; L, HyEph3). Scale bars: (A-G, I-K) $100 \mu \mathrm{m}$; (H, L) 50 um.

insulin receptor was taken as an outgroup. The result (Fig. 2A) indicates that the separation into EphAs and EphBs happened only in the vertebrate lineage and further expansion of those occurred before vertebrates diverged into their clades. The EphRs of the urochordate Ciona intestinales, the echinodermate Strongylocentrotus purpuratus, Drosophila melanogaster, Caenorhabditis elegans, Hydra vulgaris and the sponge Ephydatia fluviatilis are more similar to the EphBs of vertebrates, suggesting that these were the original EphRs. In some of the non-vertebrate lineages including Hydra an independent expansion of the EphB-family occurred.

For ephrin ligands sequences encoding the receptor-binding domains were used and the ligand binding domain of EphA1 from Mus musculus constitutes the outgroup. The result (Fig. 2B) shows that ephrins also split into ephrinAs and ephrinBs just before the divergence of the vertebrate lineage and further expansions occurred before the divergence of vertebrate clades. Invertebrate ephrin sequences are found expanded independently in each phylum and all of them are clearly separate from vertebrate sequences.

The identification of at least four different EphRs and three class B ephrin ligands in the pre-bilaterian and cnidarian Hydra indicates that this pathway evolved early in metazoans. For a long time the phylogeny of the EphR/ephrins was not clear. Bioinformatic analysis suggested that EphB receptors had evolved earlier, however, this could not be confirmed for the ligands. The four ligands of $C$. elegans possess a GPI-anchoring signal, but according to phylogenetic analysis they are more similar to class B ephrins ((Drescher, 2002) and Fig. 2B). Our data strengthen the idea that class $B$ ligands and receptors are the evolutionary older ones and that GPI-anchoring must have been invented or got lost several times.

\section{In situ hybridisations}

In situ hybridisation shows that all of the EphRs are strongly expressed in the endoderm of the tentacles (Fig. $3 \mathrm{~A}-\mathrm{L}$ ). No expression could be detected in the endo- or ectodermal cells of the body column or in nerve cells.

HyEph1 is also strongly expressed during late stages of head regeneration (36 to 48 hours, Fig. $3 \mathrm{M}-\mathrm{Q}$ ). No such upregulation was found for any of the other HyEphRs or HyEphrins. HyEph1, HyEph2 and HyEph4 additionally are strongly expressed in a 

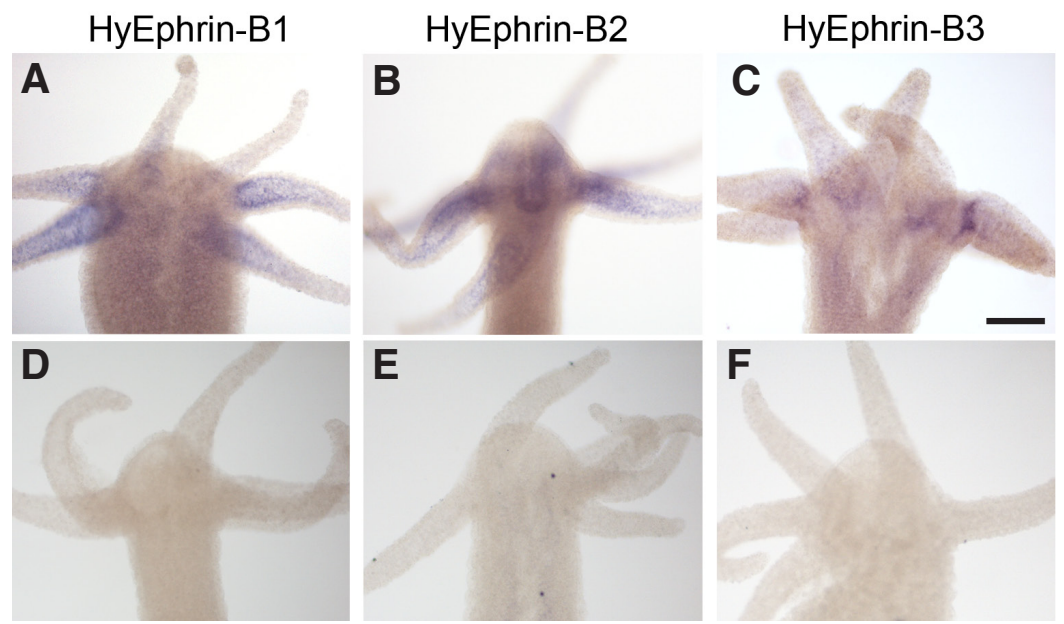

or bud tentacles. This becomes obvious in Fig. 6 where the signals at the base of the bud and in the tentacles of the bud can be compared directly.

Taken together, the expression patterns found in the tentacles as well as in the bud somewhat resemble each other (Fig. 7, schematic drawing). The receptor is expressed in endodermal cells of the tentacle and the bud. Reciprocally to this, the ephrin ligand in the late bud is expressed at the base. In the tentacles expression of ligand and receptor show more overlap than in buds, but also here ligand expression is stronger at the base of the tentacle. This graded expression and the apparent restriction of HyEphrin/HyEphR expression to appendages suggest that they could function in cell adhesion (as reviewed in (Kullander and Klein, 2002)). Epithelial cell adhesion to the mesoglea undergoes considerable changes during cell movements into tentacles and

Fig. 5. Whole-mount in situ hybridisation for the ephrin ligands HyEphrin-B1, HyEphrin-B2, HyEphrin-B3 on adult animals. NBT/BCIP (Roche) was used for staining reactions (blue signals). (A-C) anti-sense; (D-F) sense. Scale bar: $100 \mu \mathrm{m}$.

developing buds (Aufschnaiter et al., 2011). Moreover, HyEphrin-B1 is strongly upregulated at the parent-bud boundary. Ephrin-signalling mediated cell sorting and/or repulsion of adjacent cells may be involved in formation

gradient-like pattern throughout the endoderm of developing buds (Fig. 3 and Fig. 4) from early stages of budding (stage 5) to very late stages (Fig. 4 A-G). The signal disappears when the bud finally detaches (data not shown) and expression only persists in endodermal cells of the tentacles.

All HyEphrins are expressed in endodermal cells in the tentacles. In contrast to the receptor pattern, expression of the ligands appears to be stronger at the base of the tentacles and is fading out towards the tips. Again there is no detectable signal in the body column or in nerve cells (Fig. $5 \mathrm{~A}-\mathrm{C}$ ). During budding HyEphrin-B1 is strongly expressed in a sharply defined band of ectodermal cells of the bud in late stages of constriction (stage 8b). This expression persists until the bud finally detaches (Fig. 6). This pattern appears to complement the graded expression of HyEphRs in the bud. Expression at the base of the bud is much stronger than in adult
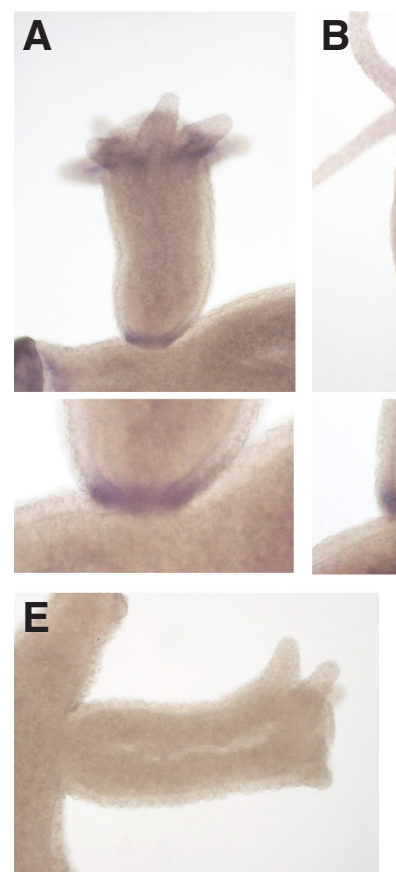
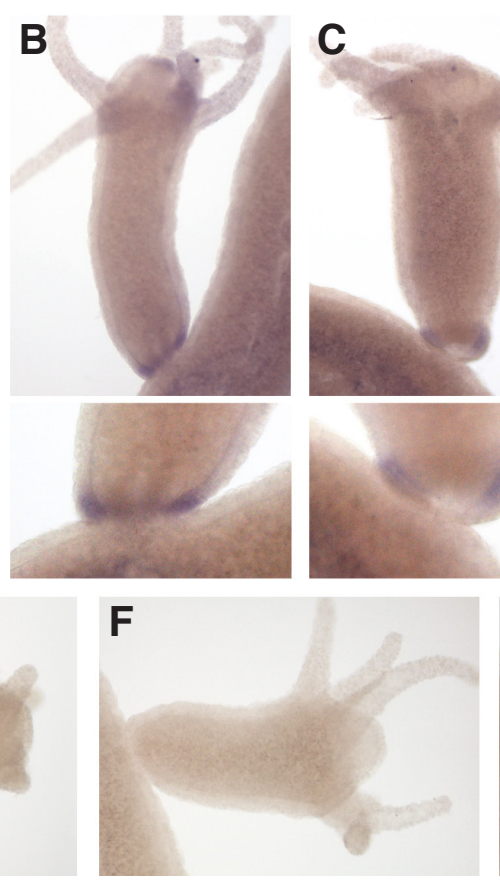
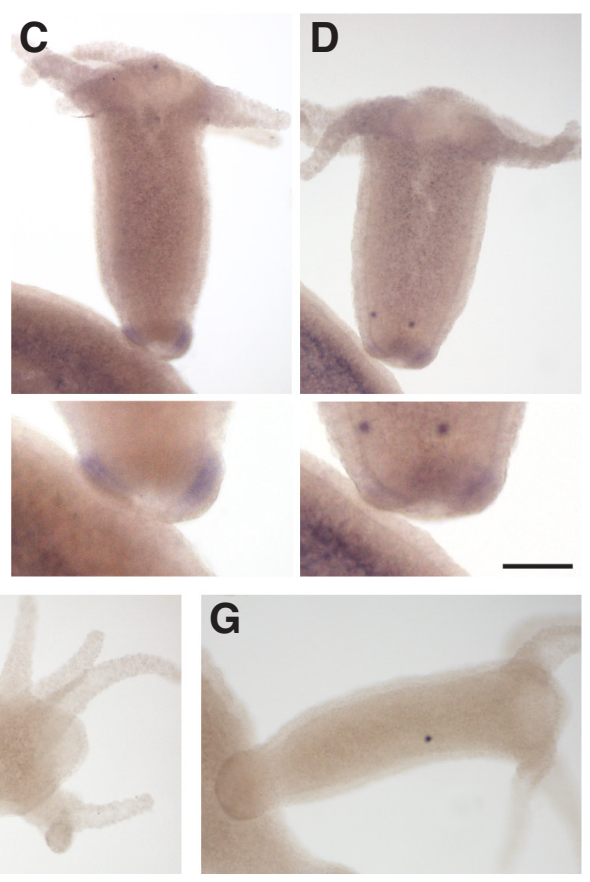
of this boundary (see Fig. 6 A-D). There also might be a link with Notch-signalling, which is important for both boundaries in Hydra (Münder et al., 2010; Münder et al., 2013).

The observed similarity of HyEphrin and HyEphR expression between tentacles and buds reminds of the observation that the tentacle and the bud axes are both characterised by apical expression of Wnts (Wnt5 at the tentacle and Wnt2/Wnt3 at the bud) and basal expression of BMP just like the main body axes of a Hydra polyp (Philipp et al., 2009), (Reinhardt et al., 2004). This lends support to the idea that tentacles are colonialized buds, as recently proposed by Meinhardt (Meinhardt, 2012). It suggests that the solitary polyps of Hydra have evolved from colonial forms, which consists of polymorphic polyps and medusa that remain attached to each other (see discussion in (Steele, 2012)).

HyEph3 is also expressed in the tentacle endoderm-cells. Furthermore it is expressed in ectodermal cells at the earliest stages of budding, where the ectoderm is getting thicker (Fig. 3 G,H and Fig. $4 \mathrm{H}$ ). Later the expression disappears. For the bud to grow out convergent extension movements of cells occur which are regulated by the planar cell polarity Wnt signalling pathway (Philipp et al., 2009). Ephrins have been shown to interact with this pathway and with Dishevelled (reviewed in (Poliakov and Wilkinson, 2006)). It is therefore possible that HyEph3 functions during cell migration at initial stages of budding.

In conclusion, our data show, that cnidarians have large families of EphR/ephrins. They support the idea that ephrin B ligands have evolved earlier and that further evolution of this protein family involved loss and gain of GPI-anchoring

Fig. 6. Whole-mount in situ hybridisation for the ephrin ligand HyEphrin-B1 on budding animals. NBT/BCIP (Roche) was used for staining reactions (blue signals). (A-D) anti-sense; (E-G) sense. Black dots in $(D, G)$ represent staining artefacts. Scale bar: $50 \mu \mathrm{m}$. 


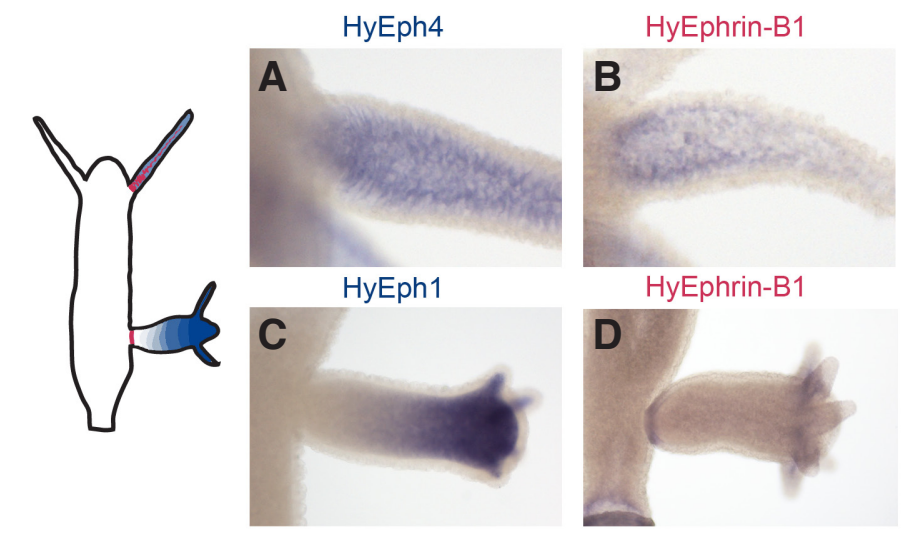

Fig. 7. Schematic overview of the expression patterns of HyEphRs and HyEphrinB ligands in the tentacle and developing bud.

of these proteins in the membranes. Their expression patterns suggest that they may participate in the regulation of epithelial cell adhesion to the mesoglea and in the formation of tissue boundaries in adult Hydra. Further studies should elucidate the precise signalling mechanism and its interaction with other signalling pathways in Hydra, especially Notch-signalling at these boundaries and the planar cell polarity Wnt-pathway during outgrowth of appendages.

\section{Materials and Methods}

\section{Hydra culture}

$H$. vulgaris strain Basel were grown in mass culture at a constant temperature of $18^{\circ} \mathrm{C}$ in hydra medium $\left(0.29 \mathrm{mM} \mathrm{CaCl}_{2}, 0.59 \mathrm{mM} \mathrm{MgSO}_{4} \mathrm{x}\right.$ $7 \mathrm{H}_{2} \mathrm{O}, 0.50 \mathrm{mM} \mathrm{NaHCO}, 0.08 \mathrm{mM} \mathrm{K}_{2} \mathrm{CO}_{3}$ ). The animals were fed regularly with freshly hatched Artemia nauplii.

\section{Cloning of EphRs and ephrin ligands}

A search for genes encoding Eph receptors and ephrin ligands was performed using the Hydra genome server (http://hydrazome.metazome. net/cgi-bin/gbrowse/hydra/) and the NCBI homepage (http://www.ncbi. nIm.nih.gov/). For the receptors, the 5' sequences (excluding TyrK and SAM domain) of all 14 receptors of Mus musculus were blasted („tBlastN“) against the Hydra genome. After comparison of all results, six gene models encoding incomplete putative EphRs were predicted, primers were designed accordingly and the fragments were cloned from Hydra vulgaris cDNA. When these sequences were blasted against a Hydra vulgaris transcriptome gene bank (kindly provided by Celina Juliano, Adrian Reich and Rob Steele) cDNA-sequences encoding 4 different full-length Eph receptors were identified. These full length Eph receptor sequences were then used to design primers for PCR amplification from Hydra cDNA and cloning into $\mathrm{pSC}$-B-amp/kana using the StrataClone ${ }^{\mathrm{TM}}$ Blunt PCR Cloning Kit (Stratagene). The receptors were named HyEph1 (XP_002155779 (5') and XP_002155898(3')), HyEph2 (XP_002161737), HyEph3 (Hma 2.228.545), HyEph4 (XP_002167854).

Genes encoding ephrin ligands were identified using the software $\mathrm{HH}$ search. A multiple sequence alignment (MSA) was built using the ProbCons software (Do et al., 2005) for 16 known ephrin protein sequences (from C. elegans: EFN-1 to -4 ; D. melanogaster, $D$. rerio: efna1a, 1b, 2 , 3a, 3b, 5a; M. musculus: efna1, efnb3; $H$. sapiens: ephrin-B2 precursor; R. norvegicus: ephrin-B1 precursor). 3 search iterations of $\mathrm{HHblits}$ (Remmert et al., 2012) were performed through the uniprot20 database to enrich the MSA with more remotely homologous ephrin sequences and computed a profile hidden Markov model (HMM) for the ephrin MSA. The same procedure was followed for every protein sequence in the Hydra genome resulting in a database of profile $\mathrm{HMMs}$, one for each hydra protein sequence. We searched with the ephrin $\mathrm{HMM}$ for ephrines in the hydra genome database using HHsearch (Remmert et al., 2012). A sequence context-specific profile for homology searching was used. Three different gene models for putative ephrin ligands were identified with HHsearch probabilities of $100 \%$ and sequence identities to $M$. musculus ephrin-B3 precursor between $18 \%$ and $25 \%$. Primers were designed and used for PCR amplification and 3' RACE experiments from Hydra cDNA. 3 Hydra ephrin ligands were cloned: HyEphrin-B1 (XP002163258 including 3'RACE), HyEphrin-B2 (Hma2.212460 (5') and Hma2.219255 (3')) and Ephrin-B3 (XP002164374) into pSC-B-amp/kana using the StrataClone ${ }^{\mathrm{TM}}$ Blunt PCR Cloning Kit (Stratagene). The EphR/ephrins in Hydra are accessible in Genbank by the numbers HyEph1 (KC751429), HyEph2 (KC751430), HyEph3 (KC751432), HyEph4 (KC751431), HyEphrin-B1 (KC751433), HyEphrin-B2 (KC751434), HyEphrin-B3 (KC751435).

\section{Domain structure analysis}

Domain structures of different protein sequences have been analysed using the SMART-software (http://smart.embl-heidelberg.de/) and big-PI, GPI-lipid anchor predictor in animals (http://mendel.imp.ac.at/sat/gpi/ gpi_server.html). Domain structures were drawn to scale via Adobe IIlustrator CS3 software.

\section{Phylogeny and gene analysis}

Neighbour joining (NJ) trees were calculated using ClustalX (ClustalX 2.0.9) multiple alignments of protein sequences (conserved tyrosine kinase domain of EphRs and receptor-binding domain of ephrins) with 10.000 bootstrap replica. The trees were displayed using NJplot. Positions with gaps had been excluded and the analysis was corrected for multiple hits (Thompson et al., 1997).

\section{Whole-mount in situ hybridisation}

Whole-mount in situ hybridisation experiments with digoxigenin labeled RNA probes were carried out as previously described (Münder et al., 2010).

\section{Acknowledgements}

The authors want to thank Celina Juliano, Rob Steele and Adrian Reich for the possibility to access unpublished transcriptome sequencing data. This work was funded by DFG-grant BO1748-5 awarded to A.B.

\section{References}

AUFSCHNAITER, R., ZAMIR, E.A., LITTLE, C.D., OZBEK, S., MUNDER, S., DAVID, C.N., LI, L., SARRAS, M.P., JR. and ZHANG, X. (2011). In vivo imaging of basement membrane movement: ECM patterning shapes Hydra polyps. J Cell Sci 124: 4027-4038.

BATLLE, E., HENDERSON, J.T., BEGHTEL, H., VANDENBORN, M.M., SANCHO, E., HULS, G., MEELDIJK, J., ROBERTSON, J., VAN DE WETERING, M., PAWSON T. et al., (2002). Beta-catenin and TCF mediate cell positioning in the intestinal epithelium by controlling the expression of EphB/ephrinB. Cell 111: 251-263.

BÖTTGER, A. and HASSEL, M. (2012). Hydra, a model system to trace the emergence of boundaries in developing eumetazoans. Int J Dev Biol 56: 583-591.

DO, C.B., MAHABHASHYAM, M.S., BRUDNO, M. and BATZOGLOU, S. (2005). ProbCons: Probabilistic consistency-based multiple sequence alignment. Genome Res 15: 330-340.

DRESCHER, U. (2002). Eph family functions from an evolutionary perspective. Curr Opin Genet Dev 12: 397-402.

GRIMMELIKHUIJZEN, C.J. and WESTFALL, J.A. (1995). The nervous systems of cnidarians. EXS 72: 7-24.

HIMANEN, J.P. and NIKOLOV, D.B. (2003). Eph signaling: a structural view. Trends Neurosci 26: 46-51.

KAO, T.J., LAW, C. and KANIA, A. (2012). Eph and ephrin signaling: lessons learned from spinal motor neurons. Semin Cell Dev Biol 23: 83-91.

KÄSBAUER, T., TOWB, P., ALEXANDROVA, O., DAVID, C.N., DALL'ARMI, E., STAUDIGL, A., STIENING, B. and BÖTTGER, A. (2007). The Notch signaling pathway in the cnidarian Hydra. Dev Biol 303: 376-390. 
KNÖLL, B. and DRESCHER, U. (2002). Ephrin-As as receptors in topographic projections. Trends Neurosci 25: 145-149.

KULLANDER, K. and KLEIN, R. (2002). Mechanisms and functions of Eph and ephrin signalling. Nat Rev Mol Cell Biol 3: 475-486.

LIMBOURG, A., PLOOM, M., ELLIGSEN, D., SORENSEN, I., ZIEGELHOEFFER, T., GOSSLER, A., DREXLER, H. and LIMBOURG, F.P. (2007). Notch ligand Delta-like 1 is essential for postnatal arteriogenesis. Circ Res 100: 363-371.

MEINHARDT, H. (2012). Modeling pattern formation in hydra: a route to understanding essential steps in development. Int J Dev Biol 56: 447-462.

MOHAMED, A.M. and CHIN-SANG, I.D. (2006). Characterization of loss-of-function and gain-of-function Eph receptor tyrosine kinase signaling in $\mathrm{C}$. elegans axon targeting and cell migration. Dev Biol 290: 164-176.

MÜNDER, S., KÄSBAUER, T., PREXL, A., AUFSCHNAITER, R., ZHANG, X., TOWB P. and BÖTTGER, A. (2010). Notch signalling defines critical boundary during budding in Hydra. Dev Biol 344: 331-345.

MÜNDER, S., TISCHER, S., GRUNDHUBER, M., BÜCHELS, N., BRUCKMEIER, N., ECKERT, S., SEEFELDT, C.A., PREXL, A., KÄSBAUER, T., BÖTTGER, A. (2013). Notch-signalling is required for head regeneration and tentacle patterning in Hydra. Dev Biol 383:146-157

ORSULIC, S. and KEMLER, R. (2000). Expression of Eph receptors and ephrins is differentially regulated by E-cadherin. J Cell Sci 113 (Pt 10): 1793-1802.

PHILIPP, I., AUFSCHNAITER, R., OZBEK, S., PONTASCH, S., JENEWEIN, M., WATANABE, H., RENTZSCH, F., HOLSTEIN, T.W. and HOBMAYER, B. (2009). $\mathrm{Wnt} /$ beta-catenin and noncanonical Wnt signaling interact in tissue evagination in the simple eumetazoan Hydra. Proc Natl Acad Sci USA 106: 4290-4295.

POLIAKOV, A. and WILKINSON, D.G. (2006). Ephrins make eyes with planar cell polarity. Nat Cell Biol 8: 7-8.

REDDY, P.C., BIDAYE, S.S. and GHASKADBI, S. (2011). Genome-wide screening reveals the emergence and divergence of RTK homologues in basal Metazoan Hydra magnipapillata. J Biosci 36: 289-296.

REINHARDT, B., BROUN, M., BLITZ, I.L. and BODE, H.R. (2004). HyBMP5-8b, a BMP5-8 orthologue, acts during axial patterning and tentacle formation in hydra. Dev Biol 267: 43-59.

REMMERT, M., BIEGERT, A., HAUSER, A. and SODING, J. (2012). HHblits: lightningfast iterative protein sequence searching by HMM-HMM alignment. Nat Methods 9: 173-175.

SIMSKE, J.S. and HARDIN, J. (2001). Getting into shape: epidermal morphogenesis in Caenorhabditis elegans embryos. Bioessays 23: 12-23.

SMALLA, M., SCHMIEDER, P., KELLY, M., TER LAAK, A., KRAUSE, G., BALL, L., WAHL, M., BORK, P. and OSCHKINAT, H. (1999). Solution structure of the receptor tyrosine kinase EphB2 SAM domain and identification of two distinct homotypic interaction sites. Protein Sci 8: 1954-1961.

STEELE, R.E. (2012). The Hydra genome: insights, puzzles and opportunities for developmental biologists. Int J Dev Biol 56: 535-542.

THOMPSON, J.D., GIBSON, T.J., PLEWNIAK, F., JEANMOUGIN, F. and HIGGINS, D.G. (1997). The CLUSTAL_X windows interface:flexible strategies for multiple sequence alignment aided by quality analysis tools. NucleicAcids Res 25: 4876-4882. 


\section{Further Related Reading, published previously in the Int. J. Dev. Biol.}

Hydra, a model system to trace the emergence of boundaries in developing eumetazoans Angelika Böttger and Monika Hassel Int. J. Dev. Biol. (2012) 56: 583-591

http://dx.doi.org/10.1387/ijdb.113454ab

Modeling pattern formation in hydra: a route to understanding essential steps in development

Hans Meinhardt

Int. J. Dev. Biol. (2012) 56: 447-462

http://dx.doi.org/10.1387/ijdb.113483hm

The Hydra genome: insights, puzzles and opportunities for Developmental Biologists Robert E. Steele

Int. J. Dev. Biol. (2012) 56: 535-542

http://dx.doi.org/10.1387/ijdb.113462rs

Cnidarians as a model system for understanding evolution and regeneration.

Brigitte Galliot and Volker Schmid

Int. J. Dev. Biol. (2002) 46: 39-48

http://dx.doi.org/10.1387/ijdb.11902686

Signals and signal-transduction systems in the control of development in Hydra and Hydractinia

M Hassel, T Leitz and W A Müller

Int. J. Dev. Biol. (1996) 40: 323-330

http://dx.doi.org/10.1387/ijdb.8735944

5 yr ISI Impact Factor $(2011)=2.959$
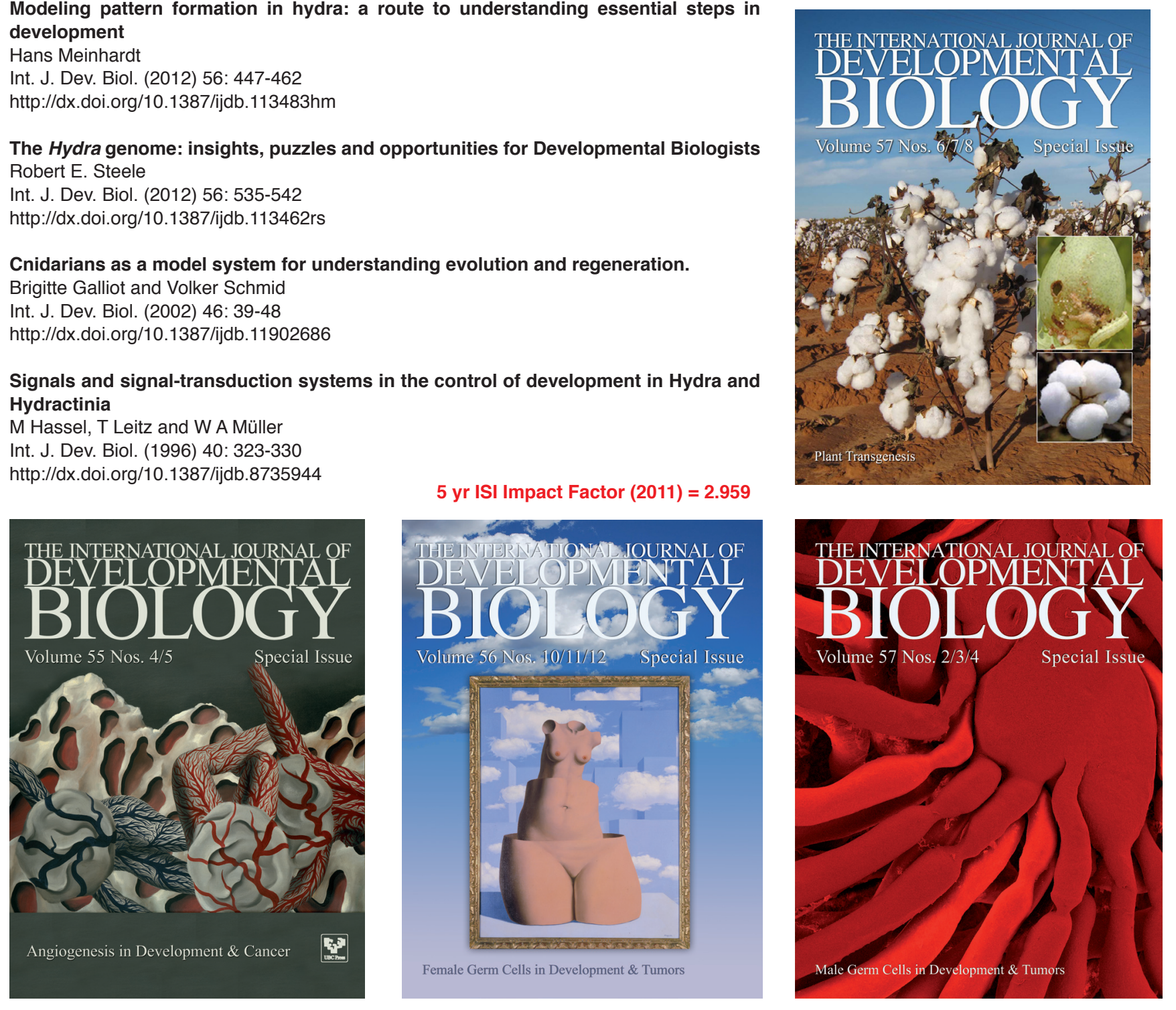